\title{
Crico-tracheal disruption and common carotid artery occlusion: a case of blunt trauma
}

A case of blum trauma to the neck is presented. While driving an all terrain vehicle (ATV), a 20-yr-old male was struck across the anterior neck by a cord suspended between two poles. Initial findings were suggestive of an isolated laryngeal injury; 48 hours laser, however, a dense left hemiplegia became manifest. A CT scan demonstrated a large right frontoparietal cerebral infarct, and an angiogram confirmed occlusion of the right commmon carotid artery. Intractable cerebral oedema developed, and the patient died five days after the initial insult. Such injuries should alert the clinician to the possibility of major vascular injury, and if suspected, angiography is warranted.

Un cas de traumatisme du cou est présenté. Tout en conduisant un véhicule tout terrain (ATV), un homme de vingt ans a été frappé à travers la face antérieure du cou par une corde suspendue entre deux poles. L'examen initial suggérait une lésion isolée du larynx; el 48 heures plus tard, cependant, une hémiplégie gauche se manifesı. Un CT scan a démontré un infarctus cérébral droit fronto-pariétale et l'angiogramme a confirmé l'acclusion de l'artère carotide commune droite. L'oedème cérébral se développa et le patient décéda cinq jours après le trauma initial. De telles lésions doivent alerter le clinicien sur la possibilité de lésions vasculaires majeures et si suspectées, l'angiographie doit être faite.

\section{Key words}

COMPLICATIONS: trauma;

ARTERIES: carotid;

LARYNX.

From the Departments of Anaesthesia, Otolaryngology, and Surgical Intensive Care, Dalhousie University and the Halifax Infirmary, 1335 Queen St., Halifax, Nova Scotia, Canada B3J $2 \mathrm{H} 6$.

Address correspondence 10: Dr. D. Oxom, Department of Anaesthesia, Sunnybrook Medical Centre, 2075 Bayview Avenue, Toronto, Ontario M4N 3M5.
Laryngeal injury is rare, occurring in less than one per cent of admissions to a major trauma centre.' Major vascular injury secondary to blunt neck trauma is also uncommon, and is more often seen with penetrating neck wounds. ${ }^{2-6}$

A case is presented in which crico-laryngeal separation secondary to a clothesline type injury (i.e., trauma to the neck at high speed by any type of horizontally suspended cord), was confirmed on surgical exploration. Two days after injury, the patient unexpectedly developed clinical evidence of a large cerebral infarct, which was confirmed by CT scan. Angiography demonstrated occlusion of the common carotid artery.

\section{Case history}

The patient, a 20 -yr-old male, presented to a peripheral hospital $24 \mathrm{hr}$ after suffering a clothesline injury to his anterior neck while driving an all terrain vehicle (ATV). All his symptoms were referable to the upper respiratory tract, and included hoarseness, dysphagia, dyspnoea, and neck swelling. Lateral $x$-rays of the neck revealed subcutaneous emphysema (Figure 1). A CT scan demonstrated crico-tracheal disruption (Figure 2), and he was immediately transferred to the operating room for tracheostomy under local anaesthesia. The trachea could not be identified, and an orotracheal tube was successfully placed with the aid of topically applied lidocaine. The patient was subsequently transferred to our institution.

On admission, the above findings were confirmed. The anterior neck was severely contused. Although sedated, the patient was moving all extremities. There was no evidence of other injury, and nothing of significance was found on cardiac, respiratory, or neurological examination. Further radiological studies included chest and cervical spine $x$-rays. Both were interpreted as being normal.

He was then taken to the operating room. Anaesthesia was induced with thiopentone, and maintained with isoflurane, intermittent boluses of fentanyl, and 100 per cent oxygen. Fibreoptic bronchoscopy showed the tracheal tube to be one inch above the carina. Through an anterior neck approach the crico-tracheal separation was repaired. 


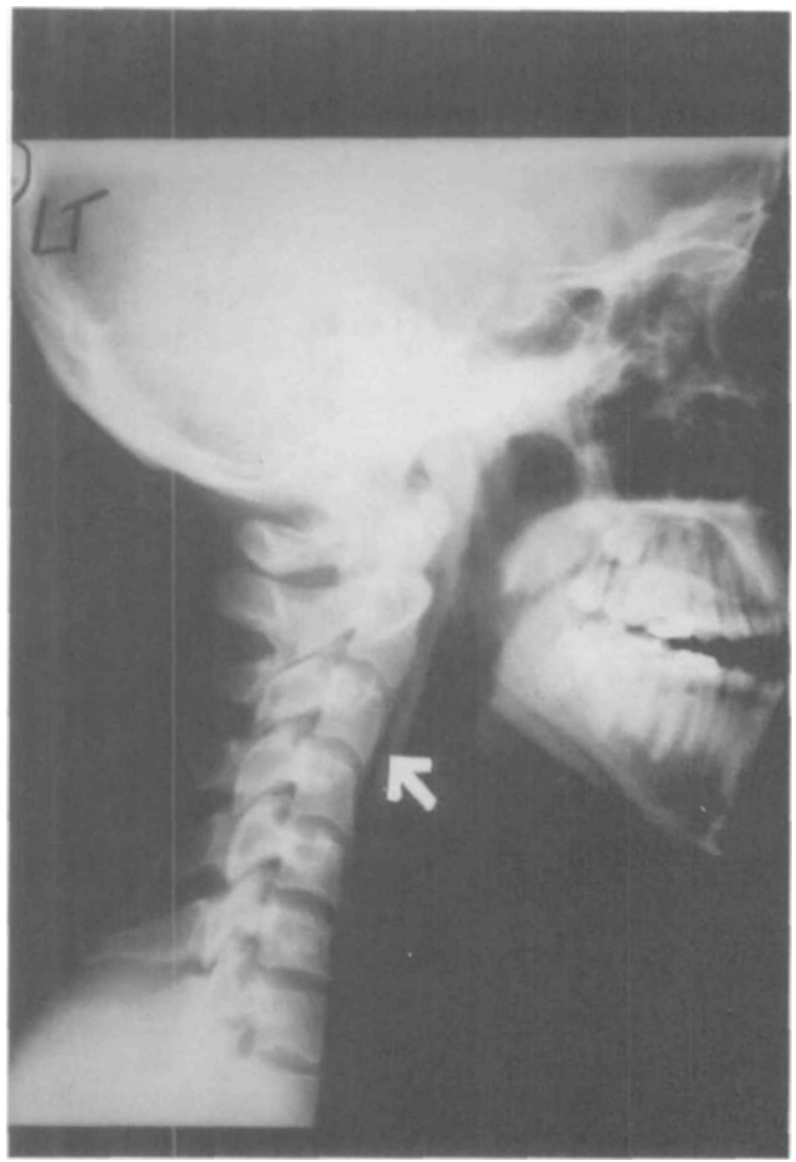

FIGURE 1 Lateral neck $x$-ray: Prevertertral air is present.

Lacerations to the epiglottis and the right arytenoid cartilage were closed through an incision in the thyrohyoid membrane. A tracheostomy was created, and the patient was returned to the surgical intensive care unit. As the patient emerged from general anaesthesia, it was noted that he had no spontaneous movement of his left arm or leg (48 hr after the initial injury). Physical examination revealed flaccid paralysis of the left-sided extremities. Pupils were equal and reactive to light.

An urgent CT scan revealed a large radiolucent area involving the right frontal and parietal lobes, consistent with a large cerebral infarct (Figure 3). Four-vessel angiography demonstrated occlusion of the right common carotid artery (Figure 4). Three days later (120 hr after initial injury), intractable cerebral oedema developed, and the patient died.

\section{Discussion}

A case is presented in which blunt trauma to the anterior neck produced airway disruption. This was clearly evi-

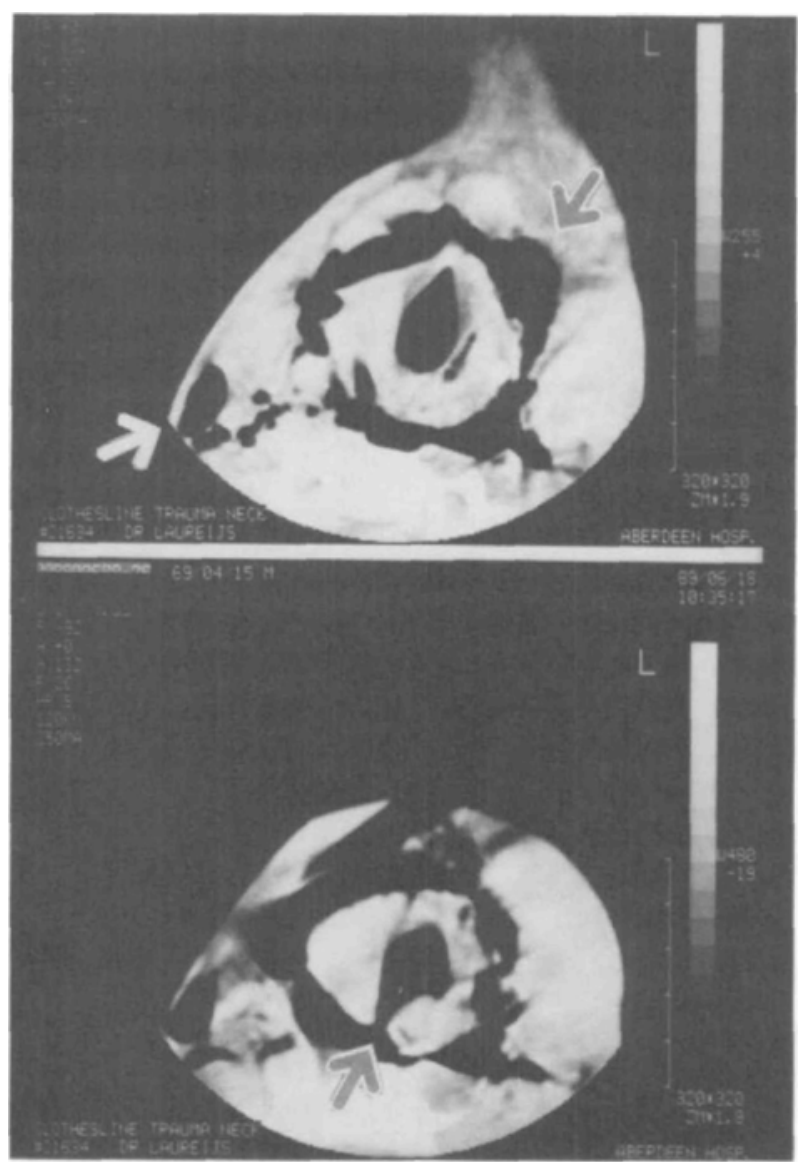

FIGURE 2 CT scan of the neck: upper panel: level of the glottis; subcutaneous and paratracheal air is present. Lower panel: level just below the cricoid cartilage; posterior defecr in the trachea is present.

dent from the history, physical examination, and radiological investigations. Occult injury to the right common carotid artery had also occurred which only became manifest 48 hours after admission. A downhill course marked by refractory cerebral oedema ensued, and the patient died five days after presentation.

The management of blunt laryngeal trauma has recently been reviewed. ${ }^{7}$ No cases of arterial injury were reported. This is consistent with what has been previously published. ${ }^{2-6}$ Routine angiography cannot therefore be recommended, and should only be performed if clinical suspicion exists. Coexisting injuries to the chest, neck, head and oesophagus may also occur, and must be ruled out.

Tracheostomy should be the only method of airway control in severe laryngotracheal trauma; ${ }^{7,8}$ this was attempted, but could not be accomplished. It was fortuitous that an orotracheal tube could be passed.

The arterial lesion was probably due to intimal damage, 


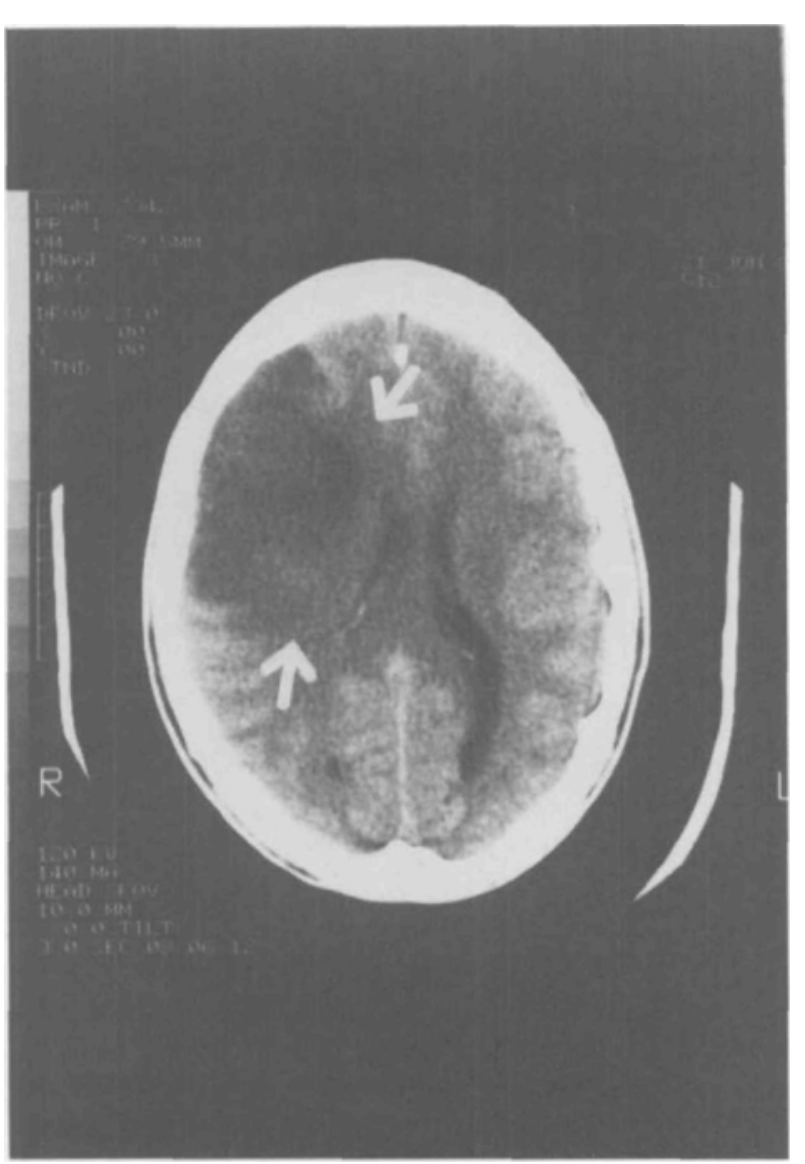

FIGURE 3 CT scan of the head. Large right fronto-parictal cercbral infarct, with compression of the lateral ventricle.

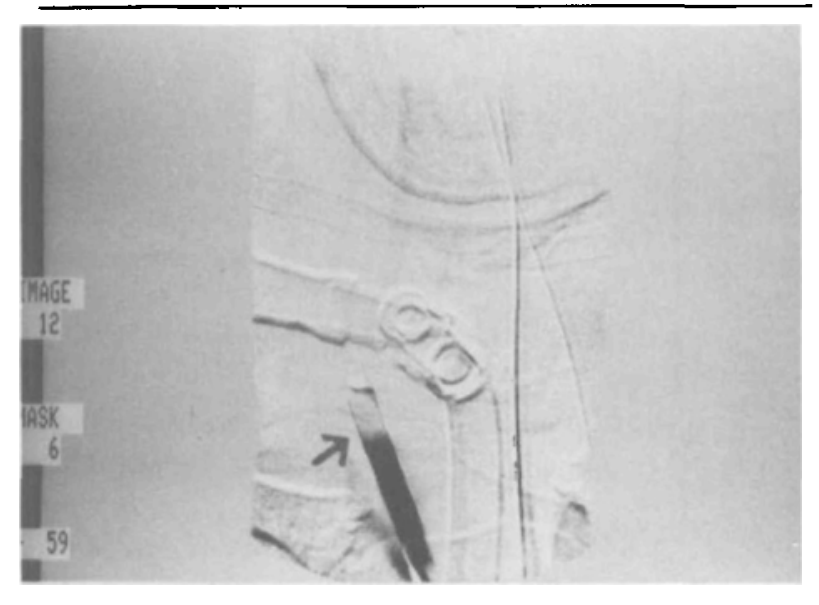

FIGURE 4 Angiogram with direct injection of the right cammon carotid artery. Obstruction to flow is present. dissection, and subsequent thrombosis. ${ }^{9}$ The mechanism may be by direct damage to the vessel, or indirectly by hyperextension and contralateral flexion of the neck and stretching of the artery. ${ }^{10}$ The time between arterial injury, and the manifestation of neurological symptoms is quite variable. With long intervals, delayed embolization may be inplicated, and it is possible that this may have occurred during the operative procedure. The incidence of arterial injury complicating blunt laryngeal trauma is unknown. In general, the prognosis of traumatic thrombosis of the carotid artery is grave. In a series of 24 cases, 85 per cent died or had major central nervous system sequelae. ${ }^{11}$

In conclusion, this case report highlights the dictum that in trauma medicine, one must diligently search for associated injuries.

\section{References}

1 Gussack AS, Jurkovich GJ. Treatment dilemunas in laryngotracheal trauma. J Trauma 1988; 28: 1439-44.

2 Herrin TJ, Brzustowicz $R$, Hendrickson $M$. Anesthetic management of neck trauma. South Med J 1979; 72 . 1102-6.

3 Cohen A, Brief D, Mathewson C. Carotid artery injuries. Am J Surg 1970; 120: 210-4.

4 Downey $W L$, Owen $R C$, Ward $P H$. Traumatic laryngeal injury - its management and sequelae. South Med J 1967; 60: 756-60.

5 Gussack GS, Jurkovich GJ, Luterman F, Gussack GS, Jurkovich GJ, Luterman A. Laryngotracheal trauma: a protocol approach to a rare injury. Laryngoscope 1986; 96 $660-5$.

6. Alonso WA, Pratt LL, Zollinger WK, Ogura JH. Complications of laryngeal disruption. Laryngoscope 1974; 84: $1276-90$.

7 Fuhrman GM, Stieg FH, Buerk CA. Blunt laryngeal trauma: classification and management protocol. J Trauma 1990; 30: 87-92.

8. Schaefer SP. Primary management of laryngeal trauma. Ann Otol-Rhinol-Laryngol 1982; 91: 399-402.

9 Davis $J M$, Zhimmerman RA. Injury of the carotid and vertebral arteries. Neuroradiology $1983 ; 25$ : 55-69.

10 Benito $M C$, Garcia F, Fernandez-Quero L et al. Lesion of the internal carotid artery caused by a car safety belt. J Trauma 1990; 30: 116-7.

11 Aarabi B, MCQueen JD. Traumatic internal carotid occlusion at the base of the skull. Surg Neurol 1978; 10 : 233-6. 